\title{
画HAD
}

DOI: http://doi.org/10.22585/hospdomic.v1i4.27

\section{Evaluación de la calidad de vida según el estado nutricional del paciente paliativo adulto: revisión sistemática}

\section{Evaluation of quality of life according to the nutritional status of the adult palliative patient: systematic review}

Ester Aracil-Lavado', Carmina Wanden-Berghe ${ }^{2,3}$, Javier Sanz-Valero', 3

1. Universidad Miguel Hernández, Elche, España.

2. Hospital General Universitario de Alicante, Alicante, España.

3. Fundación para el Fomento de la Investigación Sanitaria y Biomédica (ISABIAL-FISABIO), Alicante, España.

Correspondencia/Correspondence

Dr. Javier Sanz-Valero

Departamento de Salud Pública e Historia de la

Ciencia, Universidad Miguel Hernández.

Campus de Sant Joan d'Alacant, Alicante,

España.

jsanz@umh.es

Recibido/Received

29.07.2017

Aceptado/Accepted

06.10 .2017

\section{Conflicto de Intereses/Competing interest} Las autoras y el autor declaran la no existencia de ningún tipo de conflicto de interés 


\section{RESUMEN}

Objetivo: Revisar la literatura científica relacionada con la calidad de vida según el estado nutricional del paciente paliativo adulto.

Método: Análisis crítico de los trabajos recuperados mediante revisión sistemática. Los datos se obtuvieron de la consulta directa y acceso, vía Internet, a las siguientes bases de datos bibliográficas del ámbito de las ciencias de la salud: MEDLINE (vía PubMed), The Cochrane Library, Scopus, Cumulative Index to Nursing and Allied Health Literature (CINHAL), Web of Science y la Literatura Latinoamericana y del Caribe en Ciencias de la Salud (LILACS). Se consideró adecuado el uso de los Descriptores "Quality of life", "Nutritional Status" y "Palliative care", utilizando los filtros: «Humans», "Adult» y «Comparative Study» o «Clinical Trial». Fecha de la búsqueda: noviembre de 2016.

Resultados: Tras aplicar los criterios de inclusión y exclusión se aceptaron 4 estudios para su revisión y análisis crítico. Al evaluar la calidad de los artículos seleccionados para la revisión mediante el cuestionario CONSORT, las puntuaciones oscilaron entre 11 y 20 sobre una puntuación máxima de 25.

Conclusiones: El seguimiento nutricional de los enfermos estaba relacionado directamente con la mejora del estado nutricional, y se correspondía con el incremento de la calidad de vida. Sería deseable utilizar cuestionarios específicos y validados para evaluar la calidad de vida según el estado nutricional que permitirán minimizar cualquier tipo de subjetividad del paciente. Serían necesarios futuros estudios, con una adecuada población, que aclaren la relación directa entre el estado nutricional y la calidad de vida en los enfermos paliativos.

Palabras clave: Calidad de vida; Estado Nutricional; Cuidados Paliativos

\section{ABSTRACT}

Objective: To review the scientific literature related to the quality of life according to the nutritional status of the adult palliative patient.

Method: Critical analysis of the documents recovered by systematic review. The data were obtained from the direct consultation and access, on the Internet, in the next bibliographic databases in the area of health sciences: MEDLINE (via PubMed), The Cochrane Library, Scopus, Cumulative Index to Nursing and Allied Health Literature (CINHAL), Web of Science and Latin American and Caribbean Literature in Health Sciences (LILACS). The use of the Descriptors "Quality of life", "Nutritional status" and "Palliative care" were considered appropriate, using the filters: "Humans", "Adults" and "Comparative Study" or "Clinical Trial". Date of search: November 2016.

Results: After applying the inclusion and exclusion criteria, 4 studies were accepted for review and critical analysis. To evaluate the quality of articles selected for review using the CONSORT questionnaire, scores ranged from 11 to 20 with a maximum score of 25 .

Conclusions: The nutritional monitoring of the patients was directly related to the improvement of the nutritional status, and corresponded to the increase of the quality of life. It would be desirable to use specific and validated questionnaires to evaluate the quality of life according to the nutritional status that will allow to minimize any type of subjectivity of the patient. Future studies, with an adequate population, would be necessary to clarify the direct relationship between nutritional status and quality of life in palliative patients.

Keywords: Quality of Life; Nutritional Status; PaIliative Care 


\section{INTRODUCCIÓN}

La última etapa de las enfermedades progresivas e incurables se ha ignorado durante mucho tiempo en investigación, sin embargo, evaluar esta fase final de la enfermedad permite conocer mejor las necesidades de los pacientes (1). Tal y como prueban los resultados obtenidos en el trabajo de Marín et al. (2), el 81\% de los pacientes con tratamiento paliativo sufren malnutrición, situación que va a repercutir en gran medida en la capacidad funcional con un aumento de complicaciones, de la tasa de infecciones y disminución de la tolerancia al tratamiento oncológico, al igual que una disminución de la calidad de vida de estos pacientes. Además, tal y como señalaba Buskermolen et al. (3), los pacientes con una pérdida de peso $\geq 5 \%$ antes de la quimioterapia sufren un deterioro del estado nutricional durante este tratamiento y podrían tener una supervivencia más corta.

Un tratamiento paliativo adecuado es mucho más que controlar los síntomas (4) y su prioridad debe ser la calidad de vida del paciente (5). El apoyo nutricional debería ser una de las intervenciones de los programas de cuidados paliativos siempre que se prevea que vaya a ser beneficioso para mantener o mejorar la calidad de vida del paciente (6-8). Ahora bien, se necesita disponer de una mayor evidencia acerca de las necesidades de los pacientes paliativos para así poder mejorar su calidad de vida (9).

Así, el objetivo de este trabajo fue, revisar la literatura científica relacionada con la calidad de vida según el estado nutricional del paciente paliativo adulto.

\section{MÉTODO}

\section{Diseño:}

Estudio descriptivo transversal y análisis crítico de los trabajos recuperados mediante revisión sistemática.

\section{Fuente de obtención de los datos:}

Los datos se obtuvieron de la consulta directa y acceso, vía Internet, a las siguientes bases de datos bibliográficas del ámbito de las ciencias de la salud: MEDLINE (vía PubMed), The Cochrane Library, Scopus, Cumulative Index to Nursing and Allied Health Literature (CINHAL), Web of Science y la Literatura Latinoamericana y del Caribe en Ciencias de la Salud (LILACS).

\section{Tratamiento de la información:}

Para definir los términos de la búsqueda se consultó el Thesaurus desarrollado por la U.S. National Library of Medicine.

Se consideró adecuado el uso de los Términos "Quality of life", "Nutritional Status" y "Palliative care", tanto como Descriptores como texto en los campos de registro del título y el resumen. La ecuación de búsqueda final se desarrolló para su empleo en la base de datos MEDLINE, vía PubMed, utilizando los filtros: «Humans», «Adult» $y$ «Comparative Study» O «Clinical Trial».

La ecuación de búsqueda final fue:

("Quality of Life"[Mesh] OR "Quality of Life"[Title/Abstract] OR "Life Quality"[Title/Abstract]) AND ("Nutritional Status"[Mesh] OR "Nutritional Status"[Title/Abstract]) AND ("Palliative Care"[Mesh] OR 
"Palliative Care"[Title/Abstract] OR "Palliative Therapy"[Title/Abstract] OR "Palliative Treatment"[Title/ Abstract] OR "Palliative Surgery"[Title/Abstract] OR "Terminal Patient"[Title/Abstract])

Esta estrategia se adaptó a cada una del resto de bases de datos consultadas. La búsqueda se realizó en noviembre de 2016 y se completó con el análisis del listado bibliográfico de los artículos que fueron seleccionados.

\section{Selección final de los artículos:}

Se escogieron para su estudio los artículos, en cualquier idioma, que cumplieron los siguientes criterios: adecuarse a los objetivos de la búsqueda (estado nutricional en el enfermo paliativo), estar publicados en revistas revisadas por pares y poder recuperar el texto completo del trabajo. Se excluyeron aquellos no realizados en humanos o que no incluyeran un resultado empírico relacionado directamente con la calidad de vida.

La selección de los artículos pertinentes se realizó de forma independiente por dos autores: MSL y JSV. Para dar por válida la inclusión de los estudios se estableció que la valoración de la concordancia entre estos autores (índice Kappa) debía ser superior al $80 \%$. Siempre que se cumpliera esta condición, las posibles discordancias se solucionaron mediante la consulta a la autora CWB y posterior consenso entre todos los autores (10).

Para valorar la calidad de los documentos seleccionados se utilizaron las directrices para la publicación de estudios observacionales CONSORT (CONsolidated Standards Of Reporting Trials) (9), que contiene un listado de 25 aspectos esenciales que deben describirse en la publicación de estos estudios. Para cada artículo seleccionado se asignó un punto por cada ítem presente (en caso de no ser aplicable no puntuaba). Cuando un ítem estaba compuesto por varios puntos, estos se evaluaron de forma independiente, dándole el mismo valor a cada uno de ellos y posteriormente se realizó un promedio (siendo éste el resultado final de ese ítem), de tal forma que en ningún caso se pudiera superar la puntuación de un punto por ítem.

\section{Extracción de los datos:}

El control de la corrección de los datos se realizó mediante dobles tablas que permitieron la detección de las desviaciones y su subsanación mediante nueva consulta de los originales.

Para determinar la actualidad de los artículos se calculó el semiperíodo de Burton-Kebler (la mediana de la edad) y el Índice de Price (porcentaje de artículo con edad inferior a los 5 años).

Los estudios se agruparon según las variables a estudio, con el fin de sistematizar y facilitar la comprensión de los resultados, considerando los siguientes datos: primer autor y año de publicación, tipo de estudio, país, población que participa en el estudio, patología que padecen los participantes, intervención nutricional en el estudio, periodo en el que se realizó el trabajo, cómo se valoró la calidad de vida y los resultados obtenidos.

\section{RESULTADOS}

Al aplicar los criterios de búsqueda descritos se recuperaron un total de 131 referencias: 93 $(70,99 \%)$ en Scopus, $14(10,69 \%)$ en Web of Science, 11 (8,40\%) en The Cochrane Library, 9 $(6,87 \%)$ en MEDLINE, $3(2,29 \%)$ en CINALH y 1 (0,76\%) en LILACS. De los obtenidos, $28(21,37 \%)$ fueron rechazados por estar duplicados en más de una base de datos bibliográfica. Tras aplicar los 
criterios de inclusión y exclusión (figura 1), de los 103 (78,63\%) trabajos restantes se aceptaron 4 estudios (3,05\%) para su revisión y análisis crítico (tabla 1).

El acuerdo sobre la pertinencia de los estudios seleccionados fue del 100\%. Los artículos elegidos presentaron una obsolescencia, según el Índice de Burton Kebler, igual a 3 años, con un Índice de Price del $75,00 \%$. Al evaluar la calidad de los artículos seleccionados para la revisión mediante el cuestionario CONSORT (tabla 2), las puntuaciones oscilaron entre 11,00 y 20,00 sobre una puntuación máxima de 25,00 ítems.

Los trabajos revisados fueron 4 ensayos clínicos controlados aleatorizados. Los artículos procedían de varios países: Países Bajos (11), Australia (12), Corea del Sur (13) y Reino Unido (14). Todos los artículos estaban redactados en inglés. El año con mayor número de artículos fue 2014 con tres publicaciones (75\%) (11-13).

La población incluida en los estudios estaba comprendida entre una media de edad de 58 y 75,5 años. La patología estudiada en todos los trabajos fue el cáncer avanzado. El tipo de cáncer más frecuentemente encontrado en los diferentes estudios fue el gastrointestinal $(12,14)$.

El periodo de seguimiento en los trabajos seleccionados oscilaba entre el tiempo que duraba la radioterapia (mínimo 3 semanas) y un mes tras finalizarla (13) y 32 meses (11).

La valoración de la calidad de vida se llevó a cabo mediante el uso del cuestionario validado EORTC QLQ-C30 (European Organization for Research and Treatment of Cancer Quality of Life), en 4 investigaciones (el 100\% de los estudios incluidos), aunque también se usaron el EuroQoL-5D (Euro Quality of Life 5 Dimensions), en 1 estudio y FAACT (Functional Assesment of Anorexia/Cachexia Therapy) en otro trabajo. No se recuperó ningún artículo que utilizara cuestionarios creados al efecto.

Figura 1. Identificación y selección de estudios
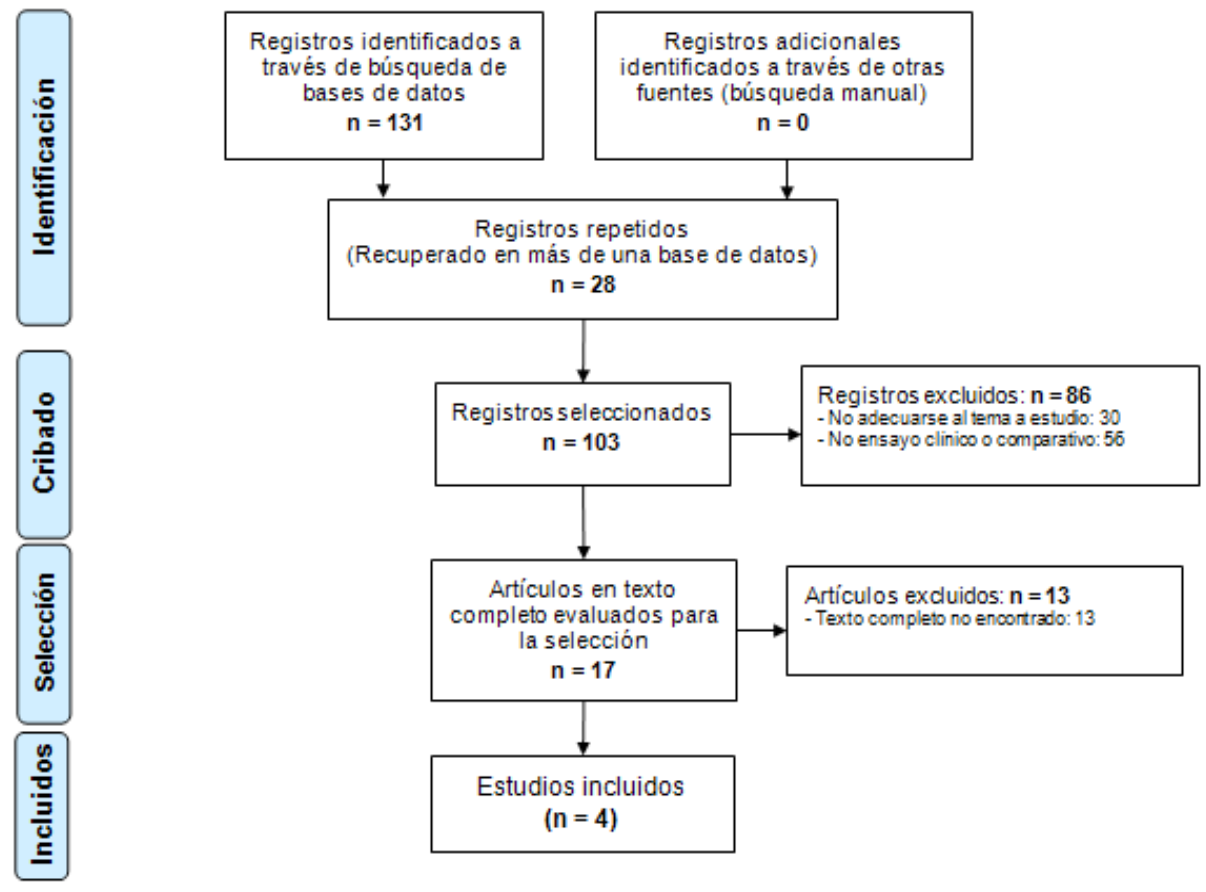


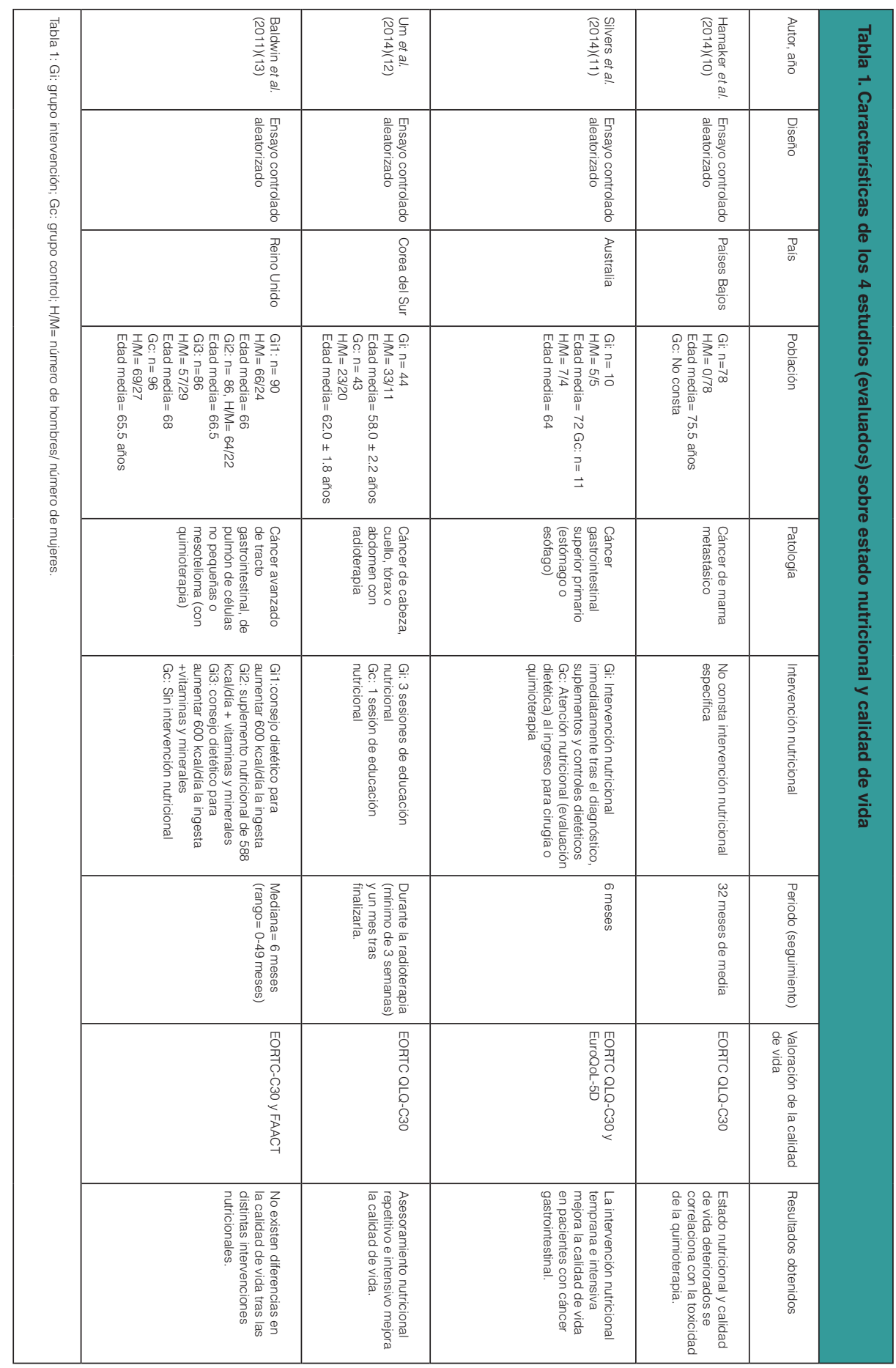


Evaluación de la calidad de vida según el estado nutricional del paciente paliativo...

\begin{tabular}{|c|c|c|c|c|}
\hline 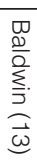 & $\frac{\stackrel{c}{3}}{\stackrel{\vec{N}}{\vec{N}}}$ & 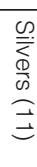 & 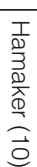 & \\
\hline- & or & - & or & - \\
\hline- & - & - & - & $N$ \\
\hline- & 0 & - & or & $\omega$ \\
\hline$\rightarrow$ & - & - & $\rightarrow$ & $\stackrel{\Delta}{\Delta}$ \\
\hline- & - & - & - & Gr \\
\hline O & or & Oे & Or & $\sigma$ \\
\hline Or & 0 & or & 0 & $v$ \\
\hline- & 0 & - & 0 & $\infty$ \\
\hline 0 & 0 & - & 0 & 0 \\
\hline- & 0 & 0 & 0 & $\overrightarrow{0}$ \\
\hline 0 & 0 & ir & 0 & $\vec{F}$ \\
\hline- & ir & 0 & or & $\overrightarrow{\mathrm{N}}$ \\
\hline- & 0 & - & - & $\vec{\omega}$ \\
\hline- & 0 & 0 & or & $\vec{A}$ \\
\hline- & - & - & - & $\vec{v}$ \\
\hline- & - & - & - & $\vec{\sigma}$ \\
\hline- & or & - & - & $\vec{v}$ \\
\hline 0 & 0 & - & 0 & $\vec{\infty}$ \\
\hline 0 & $\rightarrow$ & 0 & - & $\overrightarrow{0}$ \\
\hline- & $\rightarrow$ & - & $\rightarrow$ & $\tilde{\gamma}$ \\
\hline- & - & - & - & 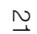 \\
\hline- & - & - & - & $\widetilde{N}$ \\
\hline- & 0 & 0 & - & $\tilde{\omega}$ \\
\hline- & 0 & 0 & $-\rightarrow$ & $\tilde{\perp}$ \\
\hline- & 0 & - & - & N \\
\hline $\begin{array}{l}\tilde{O} \\
0\end{array}$ & $\overrightarrow{\overrightarrow{0}}$ & $\begin{array}{l}\vec{v} \\
0\end{array}$ & $\begin{array}{l}\vec{\sigma} \\
\dot{\sigma}\end{array}$ & 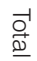 \\
\hline $\begin{array}{l}\text { : } \\
\circ\end{array}$ & $\underset{\circ}{\stackrel{f}{\circ}}$ & $\begin{array}{l}\text { \& } \\
\text { ○ }\end{array}$ & $\begin{array}{l}\text {. } \\
\circ\end{array}$ & 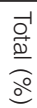 \\
\hline
\end{tabular}


La intervención nutricional realizada en los diferentes estudios fue heterogénea. Un trabajo comparó la atención nutricional al ingreso o antes de la quimioterapia con una intervención nutricional intensiva y proporcionada inmediatamente tras el diagnóstico (12). Otro estudio proporcionó al menos tres sesiones de asesoramiento dietético individualizado frente a una única sesión de educación nutricional (13). Otro artículo asesoró para aumentar la ingesta 600 kcal/día a un grupo, a un segundo grupo le proporcionó un suplemento nutricional de $588 \mathrm{Kcal} /$ día junto con vitaminas y minerales y a un tercer grupo se le dio consejo dietético para aumentar la ingesta 600 kcal/día además de vitaminas y minerales (14). En uno de los trabajos no constaba intervención nutricional específica (11).

En los artículos revisados se observó que las intervenciones nutricionales se relacionaban directamente con la calidad de vida y el estado nutricional en 3 de ellos (11-13) y 1 investigación concluía que no existía una relación clara entre calidad de vida e intervenciones nutricionales simples (14).

\section{DISCUSIÓN}

Con el análisis de la baja obsolescencia de los estudios incluidos queda demostrada la plena vigencia y actualidad, así como el interés del tema escogido $(15,16)$; los datos obtenidos (Índice de Price y de Burton Kebler) indican menor obsolescencia que los datos usuales en el ámbito de las ciencias de la salud, lo que denota que se trata de un área de conocimiento en plena emergencia.

El diseño de los estudios revisados indica una alta evidencia al tratarse de ensayos clínicos, según el grado de evidencia y recomendación de la U.S. Preventive Services Task Force (USPSTF) (17). El ceñir la revisión a ensayos clínicos y estudios comparativos se debió a la búsqueda de una consistente relación causa-efecto, ya que el vínculo existente entre el estado nutricional y la calidad de vida se está convirtiendo en una cuestión importante no sólo en los pacientes oncológicos, sino también en otras patologías e intervenciones (18).

El alto número de artículos no pertinentes descartados tras la búsqueda se debió principalmente a los resultados obtenidos en Web of Science y Scopus. Estas bases de datos no disponen de thesaurus (vocabulario controlado de indización), lo que quiere decir que la consulta se realiza en formato texto interrogando los campos de registro del título, resumen y palabras clave, impidiendo el uso de Descriptores, lo que se traduce en un alto "ruido" documental, ya observado anteriormente en otras revisiones sistemáticas (19). Además, al tratarse de un tema complejo la relación entre intervención nutricional y calidad de vida, se han encontrado estudios que tratan de evaluar esta relación de una manera indirecta (20-24) (con acetato de megestrol, grelina y otras combinaciones farmacológicas) que en el análisis detenido del contenido de los mismos se han tenido que rechazar por no responder a la pregunta de investigación planteada en esta revisión.

La evaluación de la calidad de los estudios incluidos en este trabajo mediante CONSORT no resultó muy elevada a pesar de tratarse de diseños robustos, de todos modos no se eliminó ningún estudio dado el bajo número de ellos.

El idioma de los estudios es el esperado, estando todos los trabajos escritos en inglés. Este idioma es el elegido para la publicación de la mayoría de los artículos ya que hacerlo en otra lengua distinta resulta negativo para la visibilidad, el factor de impacto y las citaciones. Además, el número de revistas anglófonas contenidas en las bases de datos actualmente es muy elevado (25).

La población incluida en los estudios era notablemente envejecida. La patología tratada en todos los trabajos seleccionados fue el cáncer avanzado en fase de tratamiento paliativo, probablemente debido a la creciente incidencia de esta enfermedad (26). En cuanto al período de seguimiento, 12 semanas, se considera un tiempo prudencial para valorar resultados (27), requisito que 
cumplen todos los estudios seleccionados excepto uno (13) en el que no queda claro el período de seguimiento.

Por otra parte, como se ha visto en los pocos estudios incluidos en la revisión, el seguimiento nutricional de los enfermos estaba relacionado directamente con la mejora del estado nutricional, y se correspondía con el incremento de la calidad de vida (28). Además, ya se comprobó que el asesoramiento nutricional mejora la calidad de vida tanto o más que la suplementación nutricional sin asesoramiento (29).

La mejora del estado de nutrición, como consecuencia de una adecuada intervención, permite reducir el número de complicaciones quirúrgicas (30), acorta el tiempo de recuperación y la duración de la estancia hospitalaria, mejora la tolerancia al tratamiento (31) e incluso aumenta la tasa de supervivencia $(32,33)$, y todo ello con una disminución general de la morbilidad (34). Ahora bien, la eficacia del asesoramiento nutricional como influencia en la calidad de vida depende de la posibilidad de adaptar la intervención a la necesidad específica de cada tipo de paciente (35), no pudiéndose perder de vista que en los estudios revisados la población era paliativa. De especial importancia es la necesidad de futuros estudios que aclaren la relación directa entre el estado nutricional y la calidad de vida en los enfermos paliativos (18).

Los resultados de esta revisión muestran que para evaluar el impacto de la intervención nutricional sobre la calidad de vida de los pacientes paliativos es práctica habitual utilizar cuestionarios validados, como ocurre en todos los estudios incluidos en esta revisión. En relación a la medición de la calidad de vida relacionada con el estado nutricional hay que tener en cuenta que se verá influenciada por la percepción del paciente, el impacto de la enfermedad, el tratamiento, las expectativas y el bienestar. Por tanto deben buscarse herramientas validadas que permitan minimizar cualquier tipo de subjetividad del paciente. En esta revisión se ha constatado el uso de estos cuestionarios, en especial el EORTC QLQ-C30. Por el contrario, la utilización de herramientas genéricas requeriría mayores tamaños poblacionales para poder conseguir resultados contrastables y en la mayoría de las ocasiones estos cuestionarios se ven afectados por problemas externos (18,36). De todos modos, el uso de herramientas validadas no garantiza la obtención de resultados generalizables, como se observó en el estudio de Baldwin et al (14).

Posibles limitaciones al estudio: La principal limitación sería el escaso número de estudios recuperados que impide tener una clara evidencia entre la intervención nutricional y la mejora, o no, de la calidad de vida.

Por todo lo anteriormente expuesto, se puede concluir: El seguimiento nutricional de los enfermos estaba relacionado directamente con la mejora del estado nutricional, y se correspondía con el incremento de la calidad de vida. Sería deseable utilizar cuestionarios específicos y validados para evaluar la calidad de vida según el estado nutricional que permitirán minimizar cualquier tipo de subjetividad del paciente. Serían necesarios futuros estudios, con una adecuada población, que aclaren la relación directa entre el estado nutricional y la calidad de vida en los enfermos paliativos.

\section{BIBLIOGRAFÍA}

1. Gainza Miranda D, Sanz Peces EM, Alonso Babarro A, Prados Sánchez MC, Varela Cerdeira M. HOLD study (Home care Obstructive Lung Disease): natural history of patients with advanced COPD. BMC Palliat Care [revista en Internet]. 2016 [citado 29 de marzo de 2017];15(1). Disponible en: http://www.biomedcentral.com/1472-684X/15/35. DOI: 10.1186/s12904-016-0104-9; PMID: 27001552 
2. Marín Caro MM, Gómez Candela C, Castillo Rabaneda R, Lourenço Nogueira T, García Huerta M, Loria Kohen V, et al. Nutritional risk evaluation and establishment of nutritional support in oncology patients according to the protocol of the Spanish Nutrition and Cancer Group. Nutr Hosp. 2008;23(5):458-68. PMID: 19160896

3. Buskermolen S, Langius JAE, Kruizenga HM, Ligthart-Melis GC, Heymans MW, Verheul HMW. Weight loss of $5 \%$ or more predicts loss of fat-free mass during palliative chemotherapy in patients with advanced cancer: a pilot study. Nutr Cancer. 2012;64(6):826-32. DOI: 10.1080/01635581.2012.690062; PMID: 22697804

4. Gagnon B, Murphy J, Eades M, Lemoignan J, Jelowicki M, Carney S, et al. A prospective evaIuation of an interdisciplinary nutrition-rehabilitation program for patients with advanced cancer. Curr Oncol. 2013;20(6):310-8. DOI: 10.3747/co.20.1612; PMID: 24311946

5. Sobotka L, Schneider SM, Berner YN, Cederholm T, Krznaric Z, Shenkin A, et al. ESPEN Guidelines on Parenteral Nutrition: Geriatrics. Clin Nutr. 2009;28(4):461-6. DOI: 10.1016/j. clnu.2009.04.004; PMID: 19464772

6. Bozzetti F, Arends J, Lundholm K, Micklewright A, Zurcher G, Muscaritoli M. ESPEN Guidelines on Parenteral Nutrition: Non-surgical oncology. Clin Nutr. 2009;28(4):445-54. DOI: 10.1016/j. clnu.2009.04.011; PMID: 19477052

7. Bozzetti F. Nutritional support of the oncology patient. Crit Rev Oncol Hematol. 2013;87(2):172200. DOI: 10.1016/j.critrevonc.2013.03.006; PMID: 23746998

8. Orrevall Y. Nutritional support at the end of life. Nutrition. 2015;31(4):615-6. DOI: 10.1016/j. nut.2014.12.004; PMID: 25770331

9. Eldridge SM, Chan CL, Campbell MJ, Bond CM, Hopewell S, Thabane L, et al. CONSORT 2010 statement: extension to randomised pilot and feasibility trials. Pilot Feasibility Stud. 2016;2:64. DOI: 10.1186/s40814-016-0105-8; PMID: 27965879

10. Wanden-Berghe C, Sanz-Valero J. Systematic reviews in nutrition: standardized methodology. $\mathrm{Br}$ J Nutr. 2012;107(S2):S3-7. DOI: 10.1017/S0007114512001432; PMID: 22591902

11. Hamaker ME, Seynaeve C, Wymenga ANM, van Tinteren H, Nortier JWR, Maartense E, et al. Baseline comprehensive geriatric assessment is associated with toxicity and survival in elderly metastatic breast cancer patients receiving single-agent chemotherapy: Results from the OMEGA study of the Dutch Breast Cancer Trialists' Group. The Breast. 2014;23(1):81-7. DOI: 10.1016/j. breast.2013.11.004; PMID: 24314824

12. Silvers MA, Savva J, Huggins CE, Truby H, Haines T. Potential benefits of early nutritional intervention in adults with upper gastrointestinal cancer: a pilot randomised trial. Support Care Cancer. 2014;22(11):3035-44. DOI: 10.1007/s00520-014-2311-3; PMID: 24908429

13. Um MH, Choi MY, Lee SM, Lee IJ, Lee CG, Park YK. Intensive nutritional counseling improves PG-SGA scores and nutritional symptoms during and after radiotherapy in Korean cancer patients. Support Care Cancer. 2014;22(11):2997-3005. DOI: 10.1007/s00520-014-2304-2; PMID: 24906838

14. Baldwin C, Spiro A, McGough C, Norman AR, Gillbanks A, Thomas K, et al. Simple nutritional intervention in patients with advanced cancers of the gastrointestinal tract, non-small cell lung cancers or mesothelioma and weight loss receiving chemotherapy: a randomised controlled trial: Oral nutritional interventions in cancer and weight loss. J Hum Nutr Diet. 2011;24(5):431-40. DOI: 10.1111/j.1365-277X.2011.01189.x; PMID: 21733143 
15. Sanz-Valero J, Wanden-Berghe C. Análisis bibliométrico de la producción científica, indizada en MEDLINE, sobre los servicios de salud proporcionados por las unidades de hospitalización a domicilio. Hosp Domic. 1(1);2017:21-34. DOI: 10.22585/hospdomic.v1i1.3

16. Quesada-Risueño P, Sanz-Valero J, Wanden-Berghe C. Análisis bibliométrico de la producción científica existente en la base de datos bibliográfica MEDLINE sobre la fibra dietética. Rev Esp Nutr Hum Diet. 2017;21:29-38. DOI: 10.14306/renhyd.21.1.275

17. Primo J. Niveles de evidencia y grados de recomendación (I/II). Enfermedad Inflamatoria Intestinal al día. 2003;2(2):39-42.

18. Wanden-Berghe C, Sanz-Valero J, Escribà-Agüir V, Castelló-Botia I, Guardiola-Wanden-Berghe R; Grupo Red Mel - CYTED. Evaluation of quality of life related to nutritional status. Br J Nutr. 2009;101(07):950-60. DOI: 10.1017/S0007114508207178; PMID: 19183508

19. Domingo-Pueyo A, Sanz-Valero J, Wanden-Berghe C. Efectos sobre la salud de la exposición laboral al cromo y sus compuestos: revisión sistemática. Arch Prev Riesgos Laborales. 2014;17(3):142-53. DOI: 10.12961/aprl.2014.17.3.03; PMID: 25022532

20. LeCaer H, Greillier L, Corre R, Jullian H, Crequit J, Falchero L, et al. A multicenter phase II randomized trial of gemcitabine followed by erlotinib at progression, versus the reverse sequence, in vulnerable elderly patients with advanced non small-cell lung cancer selected with a comprehensive geriatric assessment (the GFPC 0505 study). Lung Cancer. 2012;77(1):97-103. DOI: 10.1016/j.lungcan.2012.02.004; PMID: 22405570

21. McMillan DC, O'Gorman P, McArdle CS. A prospective randomized study of megestrol acetate and ibuprofen in gastrointestinal cancer patients with weight loss. Br J Cancer. 1999;79(3/4):495500. DOI: 10.1038/sj.bjc.6690077; PMID: 10027319

22. Lundholm K, Gunnebo L, Körner U, Iresjö B-M, Engström C, Hyltander A, et al. Effects by daily long term provision of ghrelin to unselected weight-losing cancer patients: A randomized double-blind study. Cancer. 2010;116(8):2044-52. DOI: 10.1002/cncr.24917; PMID: 20186829

23. Lindholm E. Effects of Recombinant Erythropoietin in Palliative Treatment of Unselected Cancer Patients. Clin Cancer Res. 2004;10(20):6855-64. DOI: 10.1158/1078-0432.CCR-04-0373; PMID: 15501962

24. Tomíska M, Tomisková M, Salajka F, Adam Z, Vorlícek J. Palliative treatment of cancer anorexia with oral suspension of megestrol acetate. Neoplasma. 2003;50(3):227-33. PMID: 12937858

25. Franco-López A. Publicar en castellano, o en cualquier otro idioma que no sea inglés, negativo para el factor de. J Negat No Posit Results. 2016;(2):65-70. DOI: 10.19230/jonnpr.2016.1.2.1005

26. Suess A, March JC, Prieto MA, Escudero MJ, Cabeza E, Pallicer A. El proceso asistencial de cáncer: necesidades y expectativas de los usuarios. Oncología. 2006;29(9):357-67.

27. Vásquez-Morales A, Sanz-Valero J, Wanden-Berghe C. Ejercicio excéntrico como recurso físico preventivo en personas mayores de 65 años: revisión sistemática de la literatura científica. Enferm Clínica. 2013;23(2):48-55. DOI: 10.1016/j.enfcli.2013.01.003; PMID: 23517747

28. Ravasco P, Monteiro Grillo I, Camilo M. Cancer wasting and quality of life react to early individualized nutritional counselling! Clin Nutr. 2007;26(1):7-15. DOI: 10.1016/j.clnu.2006.10.005; PMID: 17166637

29. Ravasco P, Monteiro-Grillo I, Marques Vidal P, Camilo ME. Impact of nutrition on outcome: a prospective randomized controlled trial in patients with head and neck cancer undergoing radiotherapy. Head Neck. 2005;27(8):659-68. DOI: 10.1002/hed.20221; PMID: 15920748 
30. Burden S, Todd C, Hill J, Lal S. Pre-operative nutrition support in patients undergoing gastrointestinal surgery. Cochrane Database Syst Rev. 2012;11:CD008879. DOI: 10.1002/14651858. CD008879.pub2; PMID: 23152265

31. Feinberg J, Nielsen EE, Korang SK, Halberg Engell K, Nielsen MS, Zhang K, et al. Nutrition support in hospitalised adults at nutritional risk. Cochrane Database Syst Rev. 2017;5:CD011598. DOI: 10.1002/14651858.CD011598.pub2; PMID: 28524930

32. Bozzetti F, Cozzaglio L, Biganzoli E, Chiavenna G, De Cicco M, Donati D, et al. Quality of life and length of survival in advanced cancer patients on home parenteral nutrition. Clin Nutr. 2002;21(4):281-8. DOI: http://dx.doi.org/10.1054/clnu.2002.0560; PMID: 12135587

33. Santarpia L, Alfonsi L, Pasanisi F, De Caprio C, Scalfi L, Contaldo F. Predictive factors of survival in patients with peritoneal carcinomatosis on home parenteral nutrition. Nutrition. 2006;22(4):35560. DOI: 10.1016/j.nut.2005.06.011; PMID: 16413750

34. Schneider SM, Veyres P, Pivot X, Soummer A-M, Jambou P, Filippi J, et al. Malnutrition is an independent factor associated with nosocomial infections. Br J Nutr. 2004;92(1):105-11. DOI: 10.1079/BJN20041152; PMID: 15230993

35. Marín Caro MM, Laviano A, Pichard C. Impact of nutrition on quality of life during cancer. Curr Opin Clin Nutr Metab Care. 2007;10(4):480-7. DOI: 10.1097/MCO.0b013e3281e2c983; PMID: 17563467

36. Baxter JP, Fayers PM, McKinlay AW. A review of the instruments used to assess the quality of life of adult patients with chronic intestinal failure receiving parenteral nutrition at home. $\mathrm{Br} \mathrm{J}$ Nutr. 2005;94(5):633-8. o ～DOI: https://doi.org/10.1079/BJN20051533; PMID: 16277762 